\title{
Fecal Microbiota Transplantation for Recurrent Clostridium difficile Infection in Patients With Multiple Comorbidities: Long-Term Safety and Efficacy Results From a Tertiary Care Community Hospital
}

\author{
Charumathi Raghu Subramanian ${ }^{\mathrm{a}, \mathrm{b}, \mathrm{g}}$, Swapna Talluria , Safi Ullah Khan ${ }^{\mathrm{a}, \mathrm{c}}$, \\ Jeffry A. Katz ${ }^{\mathrm{d}}$, Michael Georgetson ${ }^{\mathrm{e}}$, Preetika Sinh ${ }^{\mathrm{e}}$ f
}

\begin{abstract}
Background: Cure rates of Clostridium difficile infection (CDI) with fecal microbiota transplant (FMT) have been promising. However, there is debate regarding success of FMT in patients with comorbidities.

Methods: Electronic chart review was done to collect data on patients who underwent FMT from January 2015 to August 2017. Charts were analyzed in November 2018 with a median follow-up of 25.4 months (interquartile range 20 - 31 months).

Results: Twenty patients underwent FMT. The primary success rate at our institution was $90 \%$ and overall success rate was $100 \%$. Six patients (43\%) had FMT failure (two early and four late).

Conclusions: This case series is a description of our center's initial experience with FMT for treatment of recurrent CDI. Our high success rate reiterates the efficacy and safety of FMT in this population including patients with comorbidities.
\end{abstract}

Keywords: Fecal microbiota transplant; Clostridium difficile infection; Inflammatory bowel disease

Manuscript submitted March 5, 2020, accepted April 29, 2020

Published online August 14, 2020

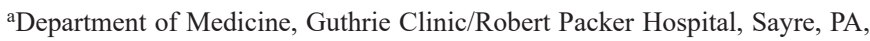
USA

${ }^{b}$ Washington Hospital Healthcare System, Fremont, CA, USA

${ }^{\mathrm{c}}$ Department of Medicine, West Virginia University, Morgantown, WV, USA

${ }^{\mathrm{d} D i v i s i o n}$ of Gastroenterology and Liver Diseases, Case Western Reserve University, University Hospitals Cleveland Medical Center, Cleveland, OH, USA eDivision of Gastroenterology, Department of Medicine, Guthrie Clinic, Sayre, PA, USA

fDivision of Gastroenterology and Hepatology, Medical College of Wisconsin, Milwaukee, WI, USA

gCorresponding Author: Charumathi Raghu Subramanian, Department of Medicine, Guthrie Clinic/Robert Packer Hospital, Sayre, PA, USA.

Email: Charumathiraghus@gmail.com

doi: https://doi.org/10.14740/gr1275

\section{Introduction}

Clostridium difficile (C. difficile) is a spore-forming, grampositive bacterium that causes infectious diarrhea and colitis. Clostridium difficile infection (CDI) has become one of the leading hospital acquired infections in the United States [1]. Exposure to hospital setting and antibiotics are known to increase the risk of CDI. However, it is becoming increasingly prevalent in the outpatient setting $[2,3]$.

CDI recurrence can occur in $15-20 \%$ of cases after the first episode and subsequent rates of recurrence are even higher (33-60\%) [4-7]. Advanced age (> 65 years), concurrent use of antibiotics for non-CDI, use of proton pump inhibitors (PPIs) and presence of comorbidities are factors associated with recurrence $[8,9]$. The rate of CDI recurrence has increased since the year 2000 and the emergence of hypervirulent strain, ribotype 027 , may have contributed to this factor [10].

CDI recurrence can be due to relapse (resumption of primary infection) or reinfection (new exposure to $C$. difficile). It can be difficult to differentiate the two in a clinical setting but the management of both is similar. The cornerstone of recurrence is reduced diversity of the microbiota and loss of resistance to colonization by opportunistic infections like $C$. difficile. Standard antibiotic treatments and recurrent episodes contribute to the development of a vicious cycle of gut dysbiosis [10].

As per the recent Infectious Disease Society of America recommendations, the treatment options for second recurrence of CDI are vancomycin with tapered or pulsed regimen, or oral vancomycin followed by fidaxomicin or rifaximin, or fecal microbiota transplantation (FMT) [11]. The aim of FMT is to restore "normal" flora to the human gut and prevent colonization of pathogenic bacteria. A study comparing fecal samples before and after FMT showed marked difference in gut bacteria after FMT. Fecal microbiota of patients after FMT was more diverse and resembled the donor microbiota rather than host microbiota prior to transplant, suggesting that the imbalance of microbiome during recurrent $\mathrm{CDI}$ is at least partially restored following FMT [12]. FMT is recommended for patients with multiple recurrences who have failed appropriate antibiotic regimens [11].

Cure rates with FMT for CDI in the general population have been reported as high as $90 \%$ [13]. Higher treatment 
failure rates in patients with comorbidities and immunocompromised state have been reported $[14,15]$. In patients with inflammatory bowel disease (IBD), there is a concern for flare in those undergoing FMT for CDI. Fischer and colleagues reported in their observational multicenter cohort study of 67 patients, a lower but reasonable success rate in IBD patients $(80 \%)$ with low rates of complications and IBD flare [16]. FMT was also effective in severely ill patients and those with multiple comorbidities although safety was a concern [17-19]. A recent study carried out in a single-center tertiary care hospital with a long-term follow-up evaluated 78 patients with and without IBD for a median period of 25 months (interquartile range (IQR) 16 - 37 months) and found no difference in the primary cure rate $(77 \%)$ and secondary cure rate $(100 \%)$ in the two groups $(27 \%$ IBD). This again suggests an overall similar cure rate in patients with comorbidities [20].

In this case series, we describe a tertiary care center's experience with FMT for recurrent CDI in patients with varied clinical background including those with comorbidities and IBD.

\section{Materials and Methods}

\section{Definitions and outcomes}

CDI was defined as presence of diarrhea ( $\geq 3$ loose stools in $24 \mathrm{~h}$ ) and stool test positive for $C$. difficile toxin by Cepheid gene Xpert infinity PCR at the local institution laboratory. Early FMT failure was defined as diarrhea and positive stool test for $C$. difficile toxin within 8 weeks [21], and recurrence/ late FMT failure was defined as diarrhea and positive test after 8 weeks. Severe CDI was defined as CDI associated with elevated serum creatinine $\geq 1.5 \mathrm{mg} / \mathrm{dL}$, and white blood cell count $>15,000 / \mathrm{mL}[11]$.

\section{Setting}

Guthrie Health System/Robert Packer Hospital, Sayre, PA, USA is a 254-bed tertiary care, community teaching hospital with a clinical microbiology laboratory that receives samples from all inpatients and outpatients of the hospital system.

\section{Inclusion criteria}

Adults $>18$ years of age with three or more recurrences of CDI who were evaluated in outpatient clinic by treating gastroenterologist (PS) were included. There were no specific exclusion criteria.

\section{FMT}

\section{Initial consultation}

Prior to scheduling FMT, a consultation was arranged with the physician performing the FMT procedure to determine appropriateness of FMT indication. At that time, a suitable donor was identified, and informed consent was obtained. A standard FMT protocol was used with modification at the study center [22].

\section{Patient and donor screening}

The donor was a person known to the patient (friend or relative), willing to participate in FMT. Donors were screened for hepatitis A, B, C, HIV, syphilis, $C$. difficile, parasites and enteric pathogens (including $E$. coli $\mathrm{O} 157: \mathrm{H} 7)$ ). The donors were excluded if they used antibiotics 3 weeks prior to the transplantation, were on anticancer drugs, suffered from chronic diarrhea, IBD, colorectal cancer, or engaged in high-risk sexual behavior. Patients were screened for hepatitis A, B, C, HIV and syphilis.

\section{Donor stool preparation}

The donor and the patient prepared stool for FMT. The donor was asked to take a capful of over-the-counter polyethylene glycol 3350 mixed with water the evening prior to the scheduled FMT. The next morning, a fistful amount of the donor's stool was collected and dissolved in $500 \mathrm{cc}$ of normal saline (provided by the hospital as part of the preparation kit). The mixture was filtered through a disposable coffee filter or strainer to eliminate particles. The fluid was collected in a clean plastic container and refrigerated in a portable cooler as it was transported to the endoscopy unit. The stool effluent was used within $6 \mathrm{~h}$ of collection.

\section{FMT procedure}

Patients had a standard colonoscopy preparation the evening prior to the examination. The colonoscopy was performed in the standard fashion, and in the right colon approximately 500 $\mathrm{mL}$ of the donor stool was instilled. Patients were instructed to take two antidiarrheal tablets prior to the procedure and hold the stool for at least $2 \mathrm{~h}$ or as long as possible after the procedure in the left lateral position. Vancomycin taper was continued till the day before FMT. Vancomycin was stopped after FMT.

\section{Follow-up consultation}

Patients were followed by the treating physician in clinic after 1 month of the procedure and subsequently based on symptoms.

\section{Data collection}

Electronic chart review was done to collect patient data in- 
Table 1. Baseline Patient Characteristics

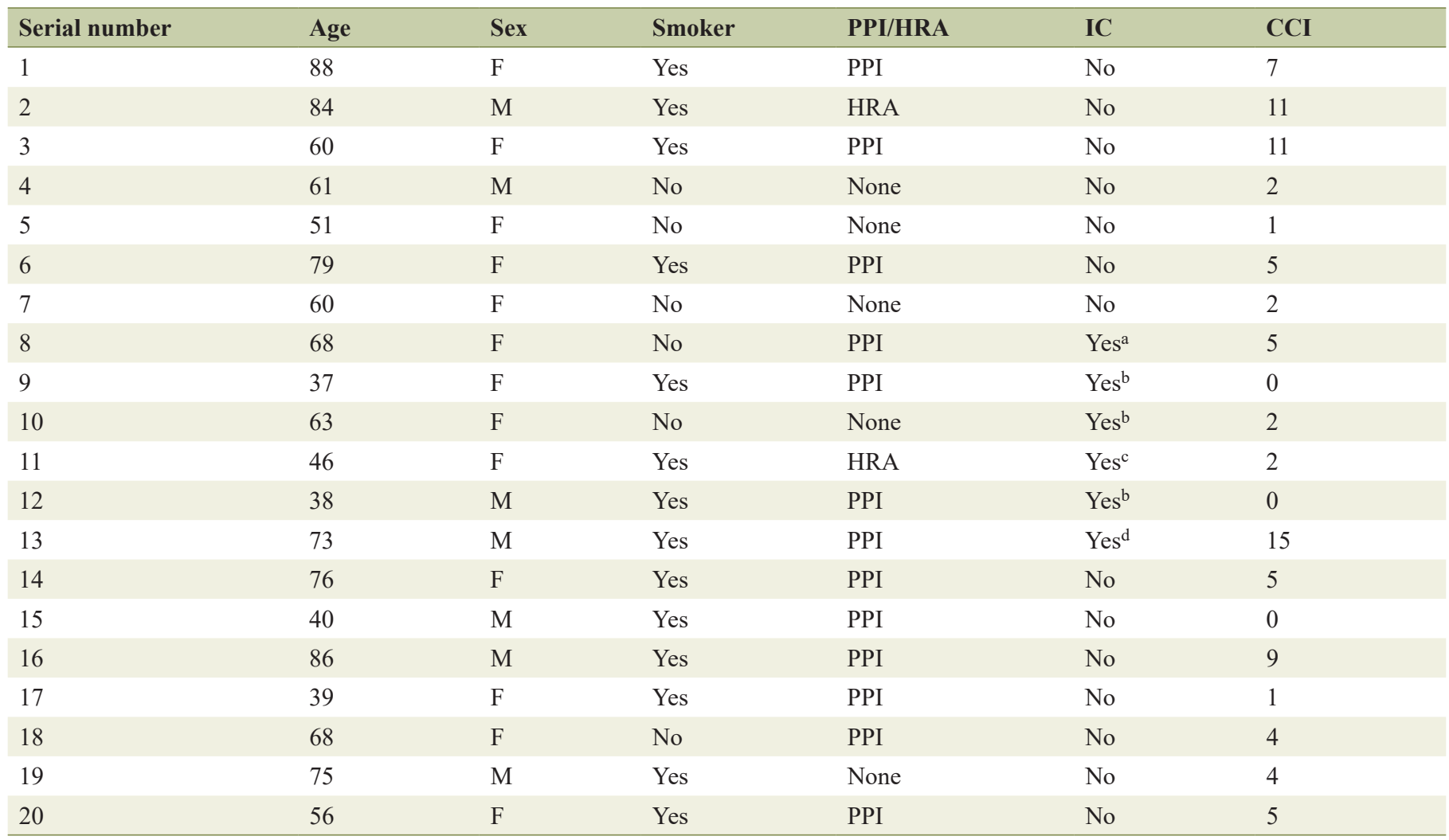

${ }^{a}$ Chemotherapy for breast cancer, non-Hodgkin lymphoma. bImmunotherapy for inflammatory bowel disease. ${ }^{c}$ Chemotherapy for breast cancer. ${ }^{d}$ Chemotherapy for lung cancer. PPI: proton pump inhibitor; HRA: histamine receptor antagonist; IC: immunocompromised; CCI: Charlson comorbidity index.

cluding demographics, severity of CDI, number of CDI recurrences, antibiotic use, comorbidities and outcomes. Data were collected on patients who underwent FMT from January 2015 to August 2017. Charts were analyzed in November 2018. The study was approved by Institutional Review Board (IRB 181064).

\section{Statistical analysis}

Descriptive analyses like frequencies and measures of central tendencies were used for continuous variables. Due to low sample size, further statistical analysis could not be performed.

\section{Results}

Twenty patients underwent FMT. Mean age was $62.4 \pm 16.57$ years with $35 \%(7 / 20)$ being male. Twelve $(60 \%)$ patients had documented prior antibiotic use before initial CDI. Fourteen (70\%) patients were active or former smokers. Thirteen $(65 \%)$ patients were on PPIs. Five (25\%) patients had an episode of severe CDI at some point. One patient (5\%) had severe sepsis with fulminant CDI refractory to oral and intravenous antibiotics along with recurrence of CDI requiring gastrojejunal van- comycin before FMT. The median follow-up period was 25.4 months (IQR 20 - 31 months).

Three patients (15\%) had end-stage renal disease (ESRD), five $(25 \%)$ had malignancy and three $(15 \%)$ had IBD, out of which two had Crohn's disease (CD) and one had ulcerative colitis (UC). Two (10\%) patients had irritable bowel syndrome (IBS) (Table 1). Out the total 20 patients, six (30\%) patients were immunosuppressed due to chemotherapy for malignancy or IBD medications.

Overall, six patients (43\%) had FMT failure (early and late). Two patients $(10 \%)$ had early FMT failure. Out of the two early failures, one patient (no. 10) with a history of UC reported improvement in frequency of bowel movements and had partial response to FMT but had positive stool test for $C$. difficile PCR within 10 days after FMT. Second patient (no. 8) had initial improvement in diarrhea but had positive stool testing with symptoms within 2 weeks after FMT. Both patients were treated with vancomycin followed by repeat FMT with successful resolution of CDI (Table 2, Fig. 1). C. difficile toxin immunoassay or glutamate dehydrogenase antigen $(\mathrm{GDH})$ testing was not available at the institution.

Four $(20 \%)$ patients had late FMT failure or recurrence of CDI after 8 weeks of FMT. All had successful resolution either with repeat FMT or antibiotic treatment during the study peri- 
Table 2. Characteristics of Patients With FMT Recurrence and Subsequent Treatment

\begin{tabular}{|c|c|c|c|c|c|c|c|}
\hline $\begin{array}{l}\text { Serial } \\
\text { number }\end{array}$ & $\begin{array}{l}\text { Antibiotic } \\
\text { use before } \\
\text { initial CDI }\end{array}$ & $\begin{array}{l}\text { Number of } \\
\text { recurrences } \\
\text { before FMT }\end{array}$ & $\begin{array}{l}\text { Antibiotics } \\
\text { used to treat } \\
\text { initial CDI }\end{array}$ & $\begin{array}{l}\text { Antibiotic } \\
\text { use before } \\
\text { recurrence }\end{array}$ & $\begin{array}{l}\text { Time of } \\
\text { recurrence } \\
\text { after FMT }\end{array}$ & $\begin{array}{l}\text { Treatment for } \\
\text { recurrence } \\
\text { after FMT }\end{array}$ & Death \\
\hline 1 & Yes & 6 & $\mathrm{M}$ & Yes & 18 months & $\mathrm{V}$ & No \\
\hline 2 & Yes & 5 & M & - & None & - & No \\
\hline 3 & Yes & 3 & $\mathrm{M}+\mathrm{V}$ & - & None & - & No \\
\hline 4 & Yes & 4 & $\mathrm{M}+\mathrm{V}$ & - & None & - & No \\
\hline 6 & Yes & 3 & $\mathrm{M}$ & - & None & - & No \\
\hline 7 & No & 3 & $\mathrm{M}$ & No & 5 months & FMT & No \\
\hline 8 & Yes & 7 & $M+V$ & No & 2 weeks & FMT & No \\
\hline 9 & Yes & 4 & $\mathrm{M}$ & - & None & - & No \\
\hline 13 & No & 4 & $\mathrm{M}$ & - & None & - & No \\
\hline 14 & Yes & 4 & $\mathrm{M}$ & - & None & - & No \\
\hline 15 & Yes & 6 & $\mathrm{M}$ & - & None & - & No \\
\hline 16 & Yes & 4 & $\mathrm{M}$ & - & None & - & Yes \\
\hline 17 & No & 4 & $\mathrm{M}$ & No & 8 months & $\mathrm{M}$ & No \\
\hline 18 & No & 4 & $M+V$ & - & None & - & No \\
\hline 19 & Yes & 2 & $M+V$ & - & None & - & No \\
\hline 20 & No & 10 & $\mathrm{~V}$ & - & None & - & Yes \\
\hline
\end{tabular}

FMT: fecal microbiota transplantation; CDI: Clostridium difficile infection; M: metronidazole; V: vancomycin.

od (Fig. 1). Option of FMT was not available for patients with recurrence after 5 months due to treating physician no longer being available at the institution. Number of comorbidities or Charlson comorbidity index (CCI) was not associated with failure of FMT (Table 3). Antibiotic use for non-CDI prior to recurrence was reported in one patient.

\section{Immunosuppression predisposes to FMT failure but FMT is successful in patients with comorbidities}

Fifty percent (3/6) of FMT failure patients were immuno- suppressed compared to $21 \%(3 / 14)$ in the primary success group. Immunosuppression was due to medications in all patients. Among the six immunosuppressed patients, two had early FMT failure, one had late failure and all of them eventually had sustained cure after repeat FMT or antibiotics.

Thirty-three percent of FMT failure patients had IBD (vs. $7 \%$ in the FMT cure group). Among the three IBD patients, one had early failure and one had late failure. All of them had sustained cure after repeat treatments. The FMT treatment and eventual resolution of CDI helped resume their treatment for IBD, thus helping the disease process.

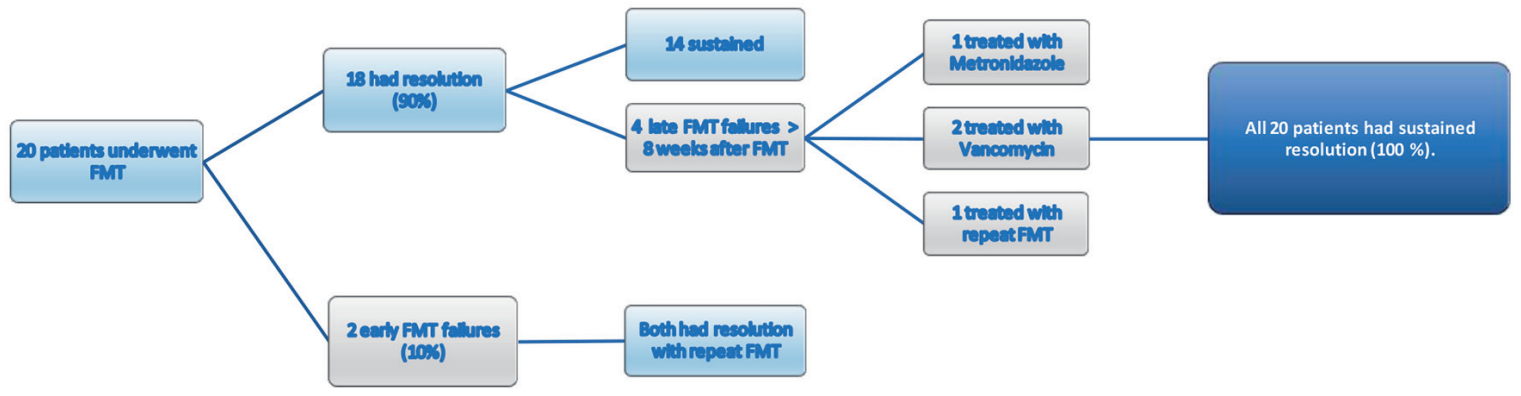

Figure 1. Response to FMT treatment. FMT: fecal microbiota transplantation. 
Table 3. Characteristics of Patients With and Without FMT Failure



FMT: fecal microbiota transplantation; PPI: proton pump inhibitor; CDI: Clostridium difficile infection; IBD: inflammatory bowel disease; CCI: Charlson comorbidity index; M: metronidazole; V: vancomycin.

\section{Patients with early failure were older, late failure had high- er PPI use}

Mean age of FMT failure patients was slightly lower than those in the primary cure group (59 vs. 64 years), but the subgroup of patients with early failure were older (65.5 years). PPI use has been reported as one of the risk factors for recurrence (43) and our study reported a slightly higher use of PPI among those with recurrence or late failure (75\% vs. $64 \%)$, but overall there was no difference between patients with failure and with FMT cure.

\section{FMT appears safe in patients with comorbidities}

None of the 20 patients reported side effects from FMT during the study period. One patient (no. 3) had nonocclusive small bowel ischemia during hemodialysis 10 days after FMT requiring exploratory laparotomy and distal jejunal resection. All-cause mortality was noted in two $(10 \%)$ patients unrelated to FMT (one patient (no. 20) died of septic shock due to pneumonia, and the other patient (no. 16) died outside our facility and hence the cause is unknown). Both deaths occurred more than 2 years after FMT.

\section{Discussion}

The human gut is a habitat of diverse group of organisms, and everyone has a unique signature of gut flora composed of 100 1,000 species of microbes, predominantly of bacterial species
$[23,24]$. In adults, the gut microbiota appears to be predominated by three clusters: the Bacteroides, the Prevotella and the Ruminococcus-dominated enterotypes [25]. Age-related changes in the microbiota with concomitant decline in the normal immune system contribute to increased risk of infection in the elderly [26].

The gut microbiota has three major functions: metabolic, protective and trophic. An alteration in the gut flora could precipitate disease states, or in contrast, could be protective against certain diseases. Gastrointestinal (GI) diseases associated with alteration in gut flora are acute diarrhea, IBS, IBD and certain malabsorptive syndromes. Evidence also suggests that altered flora may initiate colon cancer [27]. Antibiotics can have short-term effects on gut flora and cause diarrhea due to overgrowth of $C$. difficile [28].

FMT was first described in the fourth century when a Chinese doctor administered feces by mouth to patients with severe diarrhea due to food poisoning, and had positive outcomes. Subsequent reports of similar success stories from 16th century Ming dynasty are found in literature [29]. More modern descriptions date back to 1958 when Eiseman and colleagues treated pseudomembranous enterocolitis with fecal enemas with improvement [30].

FMT can be performed with a related or unrelated donor. Studies have not shown significant differences in the results between the two [31], although the possibility of better response with super donors has been reported [32]. FMT can be administered via upper GI (nasogastric, nasoenteric, or upper endoscopy) or lower GI (colonoscopy or enema) routes. The first randomized controlled trial comparing FMT and standard of care was with nasoduodenal route [33]; however, there have been subsequent trials via colonoscopy. All except one dem- 
onstrated superiority of FMT over standard of care for recurrent CDI [34-37]. A recent meta-analysis did not demonstrate significant differences in efficacy or safety between different routes of administration (upper vs. lower), and between frozen or fresh samples. The choice of delivery depends on local availability, staff expertise and patient safety and convenience [38].

Cure rates of CDI with FMT have been promising [33]. However, there has been debate regarding success of FMT in patients with comorbidities [16, 20]. We analyzed results of patients undergoing FMT for recurrent CDI in a tertiary care center with FMT via colonoscopy being performed for the first time in the institution. We compared patients with FMT failure and cure. There has been no definite classification of FMT failure or recurrence in literature [39-42] and the 8-week period of early failure was extrapolated from data describing CDI patients on antibiotics and not FMT [21, 43]. Allegretti and colleagues remarked that patients with early FMT failure could be phenotypically different from those with late failure. They proposed categorizing them separately when interpreting results [21]. Their study categorized patients into primary nonresponders (failure within 1 week), and secondary nonresponders who were in turn divided into early (between 1 and 4 weeks) and late ( $>4$ weeks). In our study, we consolidated patients into broader categories (early failure $<8$ weeks and late failure $>8$ weeks) after reviewing several classifications. As mentioned earlier, it is clinically not possible to differentiate reinfection from recurrence and the management for both is the same. Hence, we have categorized CDI occurring after 8 weeks as one group, namely, late failure or recurrence. Primary success rate in our institution was 90\% (18/20) (Fig. 1) and overall success rate was $100 \%$ over a long-term median follow-up period of 25.4 months which is comparable to the rates reported in other studies [16, 18, 44, 45].

We found that immunosuppression increased the risk of FMT failure; however, with a second treatment those patients were able to achieve a sustained response. Previous studies have also reported the need for multiple FMTs for immunosuppressed patients, as was required in our study [46, 47]. We did not find a difference in CCI among patients with and without recurrence.

FMT was well tolerated in our group. None of the patients had adverse events related to FMT. One of the reasons immunosuppressed individuals have been excluded from trials in the past is the potential risk for adverse events. Our case series reports that FMT is safe in this population. The side effects reported in other trials and systematic reviews include minor events such as abdominal discomfort, bloating, flatulence, transient fever, etc. and more serious events such as pneumonia, IBD flares, peritonitis, complications related to endoscopy and sedation $[48,49]$. Little information is available regarding long-term effects of FMT, including onset of latent infections, diabetes, IBD, colon cancer, IBS, asthma, obesity, parkinsonism or Alzheimer's. Long-term immunologic effects of FMT is also a concern, and the ideal period for follow-up after FMT for adverse events is not known. Hence, recipients of FMT, especially patients with IBD and immunosuppression need to be informed of these potential side effects and risks before the procedure [48-50]. While post-FMT IBD flares have been re- ported in other studies [47], our series did not report any flares. However, the potential risk of IBD flare needs to be discussed prior to the procedure.

It has to be noted that this is a study with significant number of patients with comorbidities including those who were immunosuppressed due to various reasons (IBD, chemotherapy, post-transplant) over a long follow-up period. Only a few studies have reported outcomes in patients with comorbidities undergoing FMT for CDI recurrence over a long-term duration $[16,20]$. This is one of the studies with longest follow-up period.

Being a retrospective and descriptive study, it has several limitations. The total number of patients is small. FMTs were done at a single center, by one gastroenterologist and in a limited time frame. Second, due to retrospective nature of the study, there are limitations related to chart review and missing data. Most patients in the area follow up in the hospital system, thus minimizing the possibility of missing outcome and safety information. Third, due to small number of patients, statistical analysis could not be performed to compare the different groups. Fourth, being a tertiary care center, there is a potential for referral bias.

In conclusion, our case series reiterates the safety and efficacy of FMT for recurrent CDI in patients with comorbidities. The long-term outcomes of FMT in patients with comorbidities seem to be promising. Prospective randomized trials evaluating FMT in CDI patients with comorbidities are needed to evaluate the implications of change in the microbiome with FMT over long term.

\section{Acknowledgments}

None to declare.

\section{Financial Disclosure}

None to declare.

\section{Conflict of Interest}

Dr Jeffry A Katz: 1) Prometheus Labs advisory board; 2) Boerhinger-Ingelheim advisory board. No disclosures for rest of the authors.

\section{Informed Consent}

Not applicable.

\section{Author Contributions}

RSC gathered data and involved in manuscript write up. ST co-wrote manuscript and helped in data acquisition. JA was involved in review of manuscript. SUK and MG helped in 
manuscript write up and review. PS performed all the FMT procedures, conceptualized the manuscript and made critical revision of the manuscript for important intellectual content.

\section{Data Availability}

The authors declare that data supporting the findings of this study are available within the article.

\section{References}

1. Miller BA, Chen LF, Sexton DJ, Anderson DJ. Comparison of the burdens of hospital-onset, healthcare facilityassociated Clostridium difficile Infection and of healthcare-associated infection due to methicillin-resistant Staphylococcus aureus in community hospitals. Infect Control Hosp Epidemiol. 2011;32(4):387-390.

2. Kelly CP, LaMont JT. Clostridium difficile - more difficult than ever. N Engl J Med. 2008;359(18):1932-1940.

3. Burke KE, Lamont JT. Clostridium difficile infection: a worldwide disease. Gut Liver. 2014;8(1):1-6.

4. Sinh P, Barrett TA, Yun L. Clostridium difficile Infection and Inflammatory Bowel Disease: A Review. Gastroenterol Res Pract. 2011;2011:136064.

5. Kelly CP. A 76-year-old man with recurrent Clostridium difficile-associated diarrhea: review of $\mathrm{C}$. difficile infection. JAMA. 2009;301(9):954-962.

6. Gerding DN, Muto CA, Owens RC, Jr. Treatment of Clostridium difficile infection. Clin Infect Dis. 2008;46(Suppl 1):S32-42.

7. Aslam S, Hamill RJ, Musher DM. Treatment of Clostridium difficile-associated disease: old therapies and new strategies. Lancet Infect Dis. 2005;5(9):549-557.

8. Deshpande A, Pasupuleti V, Thota P, Pant C, Rolston DD, Hernandez AV, Donskey CJ, et al. Risk factors for recurrent Clostridium difficile infection: a systematic review and meta-analysis. Infect Control Hosp Epidemiol. 2015;36(4):452-460.

9. Kelly CP. Can we identify patients at high risk of recurrent Clostridium difficile infection? Clin Microbiol Infect. 2012;18(Suppl 6):21-27.

10. Zanella Terrier MC, Simonet ML, Bichard P, Frossard JL. Recurrent Clostridium difficile infections: the importance of the intestinal microbiota. World J Gastroenterol. 2014;20(23):7416-7423.

11. McDonald LC, Gerding DN, Johnson S, Bakken JS, Carroll KC, Coffin SE, Dubberke ER, et al. Clinical practice guidelines for Clostridium difficile infection in adults and children: 2017 update by the Infectious Diseases Society of America (IDSA) and Society for Healthcare Epidemiology of America (SHEA). Clin Infect Dis. 2018;66(7):e1e48.

12. Seekatz AM, Aas J, Gessert CE, Rubin TA, Saman DM, Bakken JS, Young VB. Recovery of the gut microbiome following fecal microbiota transplantation. mBio. 2014;5(3):e00893-00814.

13. Bakken JS, Borody T, Brandt LJ, Brill JV, Demarco DC,
Franzos MA, Kelly C, et al. Treating Clostridium difficile infection with fecal microbiota transplantation. Clin Gastroenterol Hepatol. 2011;9(12):1044-1049.

14. Berg AM, Kelly CP, Farraye FA. Clostridium difficile infection in the inflammatory bowel disease patient. Inflamm Bowel Dis. 2013;19(1):194-204.

15. Khoruts A, Rank KM, Newman KM, Viskocil K, Vaughn BP, Hamilton MJ, Sadowsky MJ. Inflammatory bowel disease affects the outcome of fecal microbiota transplantation for recurrent Clostridium difficile infection. Clin Gastroenterol Hepatol. 2016;14(10):1433-1438.

16. Fischer M, Kao D, Kelly C, Kuchipudi A, Jafri SM, Blumenkehl M, Rex D, et al. Fecal microbiota transplantation is safe and efficacious for recurrent or refractory Clostridium difficile infection in patients with inflammatory bowel disease. Inflamm Bowel Dis. 2016;22(10):2402-2409.

17. Agrawal M, Aroniadis OC, Brandt LJ, Kelly C, Freeman S, Surawicz C, Broussard E, et al. The Long-term Efficacy and Safety of Fecal Microbiota Transplant for Recurrent, Severe, and Complicated Clostridium difficile Infection in 146 Elderly Individuals. J Clin Gastroenterol. 2016;50(5):403-407.

18. Friedman-Korn T, Livovsky DM, Maharshak N, Aviv Cohen N, Paz K, Bar-Gil Shitrit A, Goldin E, et al. Fecal transplantation for treatment of Clostridium difficile infection in elderly and debilitated patients. Dig Dis Sci. 2018;63(1):198-203.

19. Lahtinen P, Mattila E, Anttila VJ, Tillonen J, Teittinen M, Nevalainen P, Salminen S, et al. Faecal microbiota transplantation in patients with Clostridium difficile and significant comorbidities as well as in patients with new indications: A case series. World J Gastroenterol. 2017;23(39):7174-7184.

20. Tabbaa OM, Aboelsoud MM, Mattar MC. Long-term safety and efficacy of fecal microbiota transplantation in the treatment of Clostridium difficile infection in patients with and without inflammatory bowel disease: a tertiary care center's experience. Gastroenterology Res. 2018;11(6):397-403.

21. Allegretti JR, Allegretti AS, Phelps E, Xu H, Fischer M, Kassam Z. Classifying fecal microbiota transplantation failure: an observational study examining timing and characteristics of fecal microbiota transplantation failures. Clin Gastroenterol Hepatol. 2018;16(11):18321833.

22. Brandt LJ, Aroniadis OC. An overview of fecal microbiota transplantation: techniques, indications, and outcomes. Gastrointest Endosc. 2013;78(2):240-249.

23. Zoetendal EG, Akkermans AD, De Vos WM. Temperature gradient gel electrophoresis analysis of 16S rRNA from human fecal samples reveals stable and host-specific communities of active bacteria. Appl Environ Microbiol. 1998;64(10):3854-3859.

24. Qin J, Li R, Raes J, Arumugam M, Burgdorf KS, Manichanh C, Nielsen T, et al. A human gut microbial gene catalogue established by metagenomic sequencing. Nature. 2010;464(7285):59-65.

25. Arumugam M, Raes J, Pelletier E, Le Paslier D, Yamada T, Mende DR, Fernandes GR, et al. Enterotypes of the hu- 
man gut microbiome. Nature. 2011;473(7346):174-180.

26. Salazar N, Valdes-Varela L, Gonzalez S, Gueimonde M, de Los Reyes-Gavilan CG. Nutrition and the gut microbiome in the elderly. Gut Microbes. 2017;8(2):82-97.

27. Rowland IR. The role of the gastrointestinal microbiota in colorectal cancer. Curr Pharm Des. 2009;15(13):15241527.

28. Aziz Q, Dore J, Emmanuel A, Guarner F, Quigley EM. Gut microbiota and gastrointestinal health: current concepts and future directions. Neurogastroenterol Motil. 2013;25(1):4-15.

29. Zhang F, Luo W, Shi Y, Fan Z, Ji G. Should we standardize the 1,700-year-old fecal microbiota transplantation? Am J Gastroenterol. 2012;107(11):1755; author reply p 1755-11756.

30. Eiseman B, Silen W, Bascom GS, Kauvar AJ. Fecal enema as an adjunct in the treatment of pseudomembranous enterocolitis. Surgery. 1958;44(5):854-859.

31. Brandt LJ, Aroniadis OC, Mellow M, Kanatzar A, Kelly C, Park T, Stollman N, et al. Long-term follow-up of colonoscopic fecal microbiota transplant for recurrent Clostridium difficile infection. Am J Gastroenterol. 2012;107(7):1079-1087.

32. Wilson BC, Vatanen T, Cutfield WS, O'Sullivan JM. The super-donor phenomenon in fecal microbiota transplantation. Front Cell Infect Microbiol. 2019;9:2.

33. van Nood E, Vrieze A, Nieuwdorp M, Fuentes S, Zoetendal EG, de Vos WM, Visser CE, et al. Duodenal infusion of donor feces for recurrent Clostridium difficile. N Engl J Med. 2013;368(5):407-415.

34. Hota SS, Sales V, Tomlinson G, Salpeter MJ, McGeer A, Coburn B, Guttman DS, et al. Oral vancomycin followed by fecal transplantation versus tapering oral vancomycin treatment for recurrent Clostridium difficile infection: an open-label, randomized controlled trial. Clin Infect Dis. 2017;64(3):265-271.

35. Dubberke ER, Lee CH, Orenstein R, Khanna S, Hecht G, Gerding DN. Results from a randomized, placebo-controlled clinical trial of a RBX2660-A microbiota-based drug for the prevention of recurrent Clostridium difficile infection. Clin Infect Dis. 2018;67(8):1198-1204.

36. Cammarota G, Masucci L, Ianiro G, Bibbo S, Dinoi G, Costamagna G, Sanguinetti M, et al. Randomised clinical trial: faecal microbiota transplantation by colonoscopy vs. vancomycin for the treatment of recurrent Clostridium difficile infection. Aliment Pharmacol Ther. 2015;41(9):835-843.

37. Kelly CR, Khoruts A, Staley C, Sadowsky MJ, Abd M, Alani M, Bakow B, et al. Effect of fecal microbiota transplantation on recurrence in multiply recurrent Clostridium difficile infection: a randomized trial. Ann Intern Med. 2016;165(9):609-616.

38. Khan MY, Dirweesh A, Khurshid T, Siddiqui WJ. Comparing fecal microbiota transplantation to standard-of-care treatment for recurrent Clostridium difficile infection: a systematic review and meta-analysis. Eur J Gastroenterol Hepatol. 2018;30(11):1309-1317.

39. Fischer M, Kao D, Mehta SR, Martin T, Dimitry J, Keshteli AH, Cook GK, et al. Predictors of early failure after fecal microbiota transplantation for the therapy of Clostridium difficile infection: a multicenter study. Am J Gastroenterol. 2016;111(7):1024-1031.

40. Debast SB, Bauer MP, Kuijper EJ, European Society of Clinical M, Infectious D. European society of clinical microbiology and infectious diseases: update of the treatment guidance document for Clostridium difficile infection. Clin Microbiol Infect. 2014;20(Suppl 2):1-26.

41. Zar FA, Bakkanagari SR, Moorthi KM, Davis MB. A comparison of vancomycin and metronidazole for the treatment of Clostridium difficile-associated diarrhea, stratified by disease severity. Clin Infect Dis. 2007;45(3):302-307.

42. Reigadas E, Olmedo M, Valerio M, Vazquez-Cuesta S, Alcala L, Marin M, Munoz P, et al. Fecal microbiota transplantation for recurrent Clostridium difficile infection: Experience, protocol, and results. Rev Esp Quimioter. 2018;31(5):411-418.

43. Surawicz CM, Brandt LJ, Binion DG, Ananthakrishnan AN, Curry SR, Gilligan PH, McFarland LV, et al. Guidelines for diagnosis, treatment, and prevention of Clostridium difficile infections. Am J Gastroenterol. 2013;108(4):478-498; quiz 499.

44. Duarte-Chavez R, Wojda TR, Zanders TB, Geme B, Fioravanti G, Stawicki SP. Early results of fecal microbial transplantation protocol implementation at a community-based university hospital. J Glob Infect Dis. 2018;10(2):47-57.

45. Bagdasarian N, Rao K, Malani PN. Diagnosis and treatment of Clostridium difficile in adults: a systematic review. JAMA. 2015;313(4):398-408.

46. Kelly CR, Ihunnah C, Fischer M, Khoruts A, Surawicz C, Afzali A, Aroniadis O, et al. Fecal microbiota transplant for treatment of Clostridium difficile infection in immunocompromised patients. Am J Gastroenterol. 2014;109(7):1065-1071.

47. Shogbesan O, Poudel DR, Victor S, Jehangir A, Fadahunsi O, Shogbesan G, Donato A. A systematic review of the efficacy and safety of fecal microbiota transplant for Clostridium difficile infection in immunocompromised patients. Can J Gastroenterol Hepatol. 2018;2018:1394379.

48. Wang JW, Kuo CH, Kuo FC, Wang YK, Hsu WH, Yu FJ, Hu HM, et al. Fecal microbiota transplantation: review and update. J Formos Med Assoc. 2019;118(Suppl 1):S23-S31.

49. Choi HH, Cho YS. Fecal Microbiota Transplantation: Current applications, effectiveness, and future perspectives. Clin Endosc. 2016;49(3):257-265.

50. Vindigni SM, Surawicz CM. Fecal microbiota transplantation. Gastroenterol Clin North Am. 2017;46(1):171185. 